\title{
Avaliação sobre laudos do exame de mamografia no Brasil: um estudo descritivo
}

\author{
Evaluation of mammography exam reports in Brazil: a descriptive study
}

Evaluación de informes de exámenes de mamografía en Brasil: un estudio descriptivo

Henrique Rodrigues de Almeida Brito ${ }^{1}$, Maria Adriely Cunha Lima1* , Berila Beatriz Dias de Almeida1, Eduardo Paulo de Souza1, Hemmely Hevelyn Maria Araújo Melo',Gabriel Guimarães Mitidieri $^{1}$, Guilherme Barreto Vasconcelos ${ }^{1}$, Julia Alves Santos ${ }^{1}$, Antônio Vinícius Pimentel Lima², Lívia Amorim Porto ${ }^{3,4}$.

\section{RESUMO}

Objetivo: Avaliar os critérios utilizados em emissões de laudos de Mamografia no Brasil entre 2015 e 2021 , a fim de averiguar seu uso para o diagnóstico de câncer mamário em estágio inicial. Métodos: Trata-se de estudo epidemiológico descritivo quantitativo, no qual os dados referentes a mamografias foram obtidos através do Sistema de Informação do Câncer (SISCAN), com delimitação ao período entre 2015 e 2021. Resultados: No período estudado, registrou-se 15.923 .115 mamografias, sendo $99,81 \%$ realizadas em pacientes do sexo feminino. Observou-se que $98 \%$ desses exames tiveram o rastreamento de câncer mamário como indicação clínica. Destes, $63 \%$ correspondiam à faixa etária de 50 a 59 anos e $93,7 \%$ à de 40 a 74 anos. A classificação BI-RADS mais frequente foi a 2, em que correspondeu a $48,5 \%$ dos laudos. Conclusão: A maioria das mamografias foram classificadas como BI-RADS 2, além de que a principal indicação do exame foi para rastreio do câncer de mama. Foi possível observar uma em mais da metade do número de mamografias durante o ano de 2020 devido a pandemia do COVID-19.

Palavras-chave: Câncer de mama, Mamografia, Detecção precoce de câncer, Programas de rastreamento.

\section{ABSTRACT}

Objective: Evaluate the criteria used in issuing mammography reports in Brazil between 2015 and 2021, in order to investigate its use for the diagnosis of early-stage breast cancer. Methods: This is a quantitative descriptive epidemiological study, in which data related to mammography were obtained through the Cancer Information System (SISCAN), delimiting the period 2015-2021. Results: During the study period, 15,923,115 mammograms were recorded, $99.81 \%$ of which were performed in female patients. It was observed that $98 \%$ of these exams had breast cancer screening as a clinical indication. Of these, $63 \%$ corresponded to the age group from 50 to 59 years old and $93.7 \%$ to the age group from 40 to 74 years old. The most frequent BIRADS classification was 2, which corresponded to $48.5 \%$ of the reports. Conclusion: Most mammograms were classified as BI-RADS 2, and the main indication of the exam was for screening for breast cancer. It was possible to observe one in more than half the number of mammograms during the year 2020 due to a COVID19 pandemic.

Keywords: Breast cancer, Mamography, Early detection of cancer, Mass screening.

\footnotetext{
1 Universidade Tiradentes, Aracaju - SE. *E-mail: mariaadrielycunha@hotmail.com

2 Universidade Federal de Sergipe, Lagarto - SE.

${ }^{3}$ Santa Casa de Misericórdia da Bahia, Salvador - BA.

${ }^{4}$ Hospital Santa Izabel, Salvador - BA.
} 


\section{RESUMEN}

Objetivo: Evaluar los criterios utilizados en la emisión de informes de mamografías en Brasil entre 2015 y 2021, con el fin de investigar su uso para el diagnóstico de cáncer de mama en estadio temprano. Métodos: Se trata de un estudio epidemiológico descriptivo cuantitativo, en el que se obtuvieron datos relacionados con la mamografía a través del Sistema de Información del Cáncer (SISCAN), delimitando el período 2015-2021. Resultados: Durante el período de estudio se registraron 15.923.115 mamografías, de las cuales el 99,81\% se realizaron en pacientes del sexo femenino. Se observó que el $98 \%$ de estos exámenes tenían como indicación clínica el cribado del cáncer de mama. De estos, el $63 \%$ correspondió al grupo de edad de 50 a 59 años y el 93,7\% al grupo de edad de 40 a 74 años. La clasificación BI-RADS más frecuente fue 2, que correspondió al $48,5 \%$ de los reportes. Conclusión: La mayoría de las mamografías se clasificaron como BIRADS 2 y la principal indicación del examen fue la detección del cáncer de mama. Fue posible observar una en más de la mitad del número de mamografías durante el año 2020 debido a la pandemia COVID-19.

Palabras clave: Cáncer de mama, Mamografía, Detección precoz del cáncer, Tamizaje masivo.

\section{INTRODUÇÃO}

O câncer de mama é uma enfermidade de importante epidemiologia, de acordo com a Organização Mundial da Saúde tal doença registra cerca de 2 milhões de novos casos por ano ao redor do mundo, sendo que no Brasil a estimativa de casos novos de câncer de mama em 2021 foi de aproximadamente 60 mil casos, ressalta-se que o câncer de mama é a principal causa de morte dentre neoplasias do sexo feminino, sendo que em 2019 foram registrados 18.068 óbitos pela doença (INSTITUTO NACIONAL DE CÂNCER (INCA), 2019a; INCA, 2020a). Em contrapartida, o câncer de mama representa 1\% de todos os cânceres em pacientes do sexo masculino, com uma incidência de 1,06 por 100.000 homens, foi apontado como causa de óbito em 227 homens durante o ano de 2019, de acordo com os dados disponíveis no Atlas de Mortalidade por Câncer (INCA, 2021).

Devido à alta morbimortalidade do câncer de mama, sabe-se que o principal fator para um bom prognóstico é sua detecção precoce. Para isso, as estratégias utilizadas são o diagnóstico precoce, através da abordagem de pessoas com manifestações clínicas iniciais da doença, e o rastreamento, em que é realizado a aplicação de teste/exame numa população sem manifestações sugestivas, nesse caso, do câncer de mama, com o objetivo de identificar alterações indicativas da doença e encaminhar os resultados para investigação diagnóstica. Para este fim, o exame padrão ouro e mais utilizado para rastreio do câncer de mama é a mamografia, a qual é realizada através do mamógrafo, equipamento de raio $X$ capaz de identificar alterações suspeitas de malignidade antes de manifestações típicas da neoplasia surgirem (INCA, 2009; INCA, 2019a; TOMAZELLI JG, et al., 2017).

De acordo com o Ministério da Saúde (MS) a mamografia deve ser realizada na rotina clínica para detecção precoce da neoplasia mamária, sendo preconizada para fins de rastreamento em mulheres assintomáticas entre 50 e 69 anos de idade a cada dois anos, além de servir como avaliação diagnóstica e monitoramento dos grupos de risco (INCA, 2009; INCA, 2019a; TOMAZELLI JG, et al., 2017). No entanto, o Colégio Brasileiro de Radiologia e Diagnóstico por Imagem (CBR), a Sociedade Brasileira de Mastologia (SBM) e a Federação Brasileira das Associações de Ginecologia e Obstetrícia (FEBRASGO) recomendam o rastreamento anual em mulheres assintomáticas entre 40 e 74 anos, pois após os 40 anos o rastreio já está associado a redução da mortalidade pelo câncer de mama, sendo que a partir dos 75 anos ele deve ser individualizado. Além disso, ele deve ser realizado, preferencialmente, pela mamografia digital (CBR, 2020; URBAN L, et al., 2012; MIGOWSKI A, et al., 2018).

Outrossim, as mulheres com alto risco para câncer de mama também apresentam individualização do rastreio, o qual nunca deve ser realizado antes dos 25 anos. Por exemplo, mulheres com risco $\geq 20 \%$ ao longo da vida, com base na história familiar, iniciam o rastreio a partir de 30 anos ou 10 anos antes da idade do diagnóstico do parente mais jovem acometido pela doença. A idade em que é realizado o rastreamento do 
câncer de mama e a periodicidade dos exames é uma estratégia dirigida de acordo com evidências conclusivas acerca da redução da mortalidade pela neoplasia, em que o benefício da realização do exame supera os riscos de danos à saúde do exame durante sua realização (CBR, 2020; URBAN L, et al., 2012; SOUSA CNS, et al., 2019).

Para classificação das imagens obtidas pelo mamógrafo se utiliza o sistema Breast Imaging and Reporting Data System (BI-RADS $\AA)$, o qual é um protocolo utilizado para padronização dos laudos de exames de imagem de mama. Os resultados obtidos pelo BI-RADS descrevem todas as alterações mamárias encontradas e as classificam em categorias, as quais contêm a faixa de risco de malignidade e a orientação das possíveis condutas a serem realizadas.

De acordo com o CBR (2005), as categorias de avaliação são divididas em cinco, na categoria 0, em que a avaliação é dita como incompleta, sendo necessário realizar um exame de imagem adicional como ultrassonografia ou comparar com exames anteriores; já na categoria 1 não há alterações na mama, sendo necessário que a paciente realize apenas a rotina, enquanto que na categoria 2 apesar na conduta ser a mesma, observa-se a presença de achados benignos na mama como cistos simples, linfonodos intramamários, nódulos sólidos de características benignas e estáveis por 2 anos, implantes e outros.

A categoria 3 apresenta achados provavelmente benignos como cistos complexos e agrupados; e nódulos circunscritos (forma oval e orientação horizontal) sugestivo de fibroadenoma, logo é necessário realizar um controle com intervalo de tempo curto, inicialmente em 6 meses e, após isso, em 12, 24 e 36 meses em relação ao primeiro exame, tais achados apresentam um risco de malignidade de até $2 \%$. Já na categoria 4 os achados já são suspeitos de malignidade e o risco aumenta de $3 \%$ a $94 \%$, sendo necessário realizar diagnóstico citohistológico ou histopatológico. Entre os achados na mamografia observa-se nódulos sólidos com características de malignidade, além disso essa categoria pode ser subdividida de acordo com a suspeita de malignidade, 4A quando baixa, 4B intermediária e 4C moderada (CBR, 2005; INCA, 2018).

Já a categoria 5 tem achados altamente sugestivos de malignidade na mamografia, sendo realizado histopatológico para confirmação da neoplasia, de modo que seja possível realizar o tratamento, entre os achados observa-se nódulos com todas as características ecográficas de suspeição, como forma irregular, margens não circunscritas, presença de halo ecogênico, sombra acústica posterior, entre outras. Nestes exames o risco de malignidade superior a $95 \%$. A categoria 6 é quando o câncer de mama já foi confirmado por histopatologia e o paciente aguarda início do tratamento (CBR, 2005; COLÉGIO AMERICANO DE RADIOLOGIA, 2021).

Devido sua grande relevância como método de rastreio para diagnóstico precoce do câncer de mama, fazse importante avaliar a empregabilidade da mamografia no Brasil. O objetivo deste estudo consistiu em avaliar o uso da mamografia, a fim de averiguar seu uso para o diagnóstico de câncer mamário em estágio inicial, além de correlacionar a classificação BI-RADS ${ }^{\circledR}$ com variáveis como idade, periodicidade, indicação clínica, alteração em linfonodos axilares e outros.

\section{MÉTODOS}

Trata-se de um estudo epidemiológico descritivo que usa o método quantitativo, no qual os dados são disponibilizados através do Sistema de Informação do Câncer (SISCAN), que reúne as informações dos sistemas do Programa Nacional de Controle do Câncer do Colo do Útero (SISCOLO) e do Programa Nacional de Controle do Câncer de Mama (SISMAMA), acessível através das informações em saúde (TABNET) por meio do Departamento de Informática do Sistema Único de Saúde do Brasil (DATASUS), o qual possibilita acesso a informações para análises da situação sanitária, para que através disso seja possível realizar programas de ações de saúde.

A avaliação do perfil dos laudos de mamografia no Brasil delimitou-se aos anos entre 2015 e 2021, com dados limitados para com o último ano, restringindo-se até a data da última atualização do portal, 20 de agosto de 2021. A variáveis utilizadas abrangiam tanto o exame quanto o paciente. 
Dentro do contexto da análise do indivíduo, foram analisadas as seguintes variantes: sexo (masculino, feminino); faixa etária (<20 anos, entre 20 e 50, entre 50 e 70, > 70 anos); escolaridade (analfabeto (a), ensino fundamental incompleto, ensino fundamental completo, ensino médio completo, ensino superior completo); e risco elevado (sim, não, não sabe).

No que tange ao exame, foram avaliadas as variáveis: exame clínico anterior (sim, não examinadas); mamografia anterior (sim, não, não sabe); periodicidade (mesmo ano, 1 ano, 2 anos, 3 anos, 4 anos ou mais); indicação clínica (mamografia diagnóstica, mamografia rastreamento); tempo entre solicitação e liberação do exame (menos de 30 dias, 31 a 60 dias, e acima de 60 dias); linfonodo axilar direito e esquerdo (normais, alterados, não visualizados);BI-RADS (categoria 0 , categoria 1 , categoria 2 , categoria 3 , categoria 4 , categoria 5, categoria 6); nódulo na requisição da mamografia (não, na mama direita, esquerda ou em ambas); e tipo de mamografia de rastreio (população alvo, população de risco elevado -história familiar, paciente já tratado de câncer de mama).

No que diz respeito aos dados, foi utilizado o programa Microsoft Office Exce ${ }^{\circledR 3}$ para armazenamento e tabulação. Apresentados sob frequência absoluta e relativa, entre as variáveis contínuas foram calculadas medidas de dispersão (desvio padrão) e de tendências central (média).

\section{RESULTADOS}

Foram registrados 15.923.115 exames de mamografia, por local de residência, no período de 2015 a 2021 no Brasil. Através da Tabela 1, é possível analisar as características dos pacientes, em que 99,81\% eram do sexo feminino e cerca de $63 \%$ tinham entre 50 e 69 anos, sendo a faixa etária que tem recomendação pelo Ministério da Saúde para o rastreamento do câncer de mama.

Tabela 1 - Exames de mamografia de acordo com as variáveis dos pacientes, 2011-2021.

\begin{tabular}{ccc}
\hline Sexo & Total & Percentual (\%) \\
\hline Masculino & 29.878 & 0,19 \\
Feminino & 15.888 .924 & 99,81 \\
\hline Idade & & \\
\hline Menores de 20 anos & 9.645 & 0,06 \\
Entre 20 e 39 anos & 536.139 & 3,37 \\
Entre 40 e 49 anos & 4.441 .221 & 27,90 \\
Entre 50 e 69 anos & 10.032 .928 & 63,02 \\
Maiores de 70 anos & 898.811 & 5,65 \\
\hline Escolaridade & & 0,01 \\
\hline Analfabeto & 1.975 & 0,09 \\
\hline Ensino fundamental incompleto & 14.156 & 0,05 \\
Ensino fundamental completo & 8.541 & 0,04 \\
Ensino médio & 6.330 & 0,01 \\
Ensino superior & 1.414 & 99,79 \\
Ignorado & 15.886 .497 & 15,66 \\
Risco elevado & & 70,63 \\
Sim & 2.492 .256 & 13,48 \\
Não & 11.243 .966 & \\
\hline Não sabe & 2.146 .222 & \\
\hline
\end{tabular}

Fonte: Brito HRA, et al., 2021.

Nota-se que aproximadamente $99,8 \%$ dos casos não tiveram o item referente a escolaridade preenchido, isso dificulta a análise se há relação entre o nível de escolaridade e a realização da mamografia (Tabela 1). Já na Tabela 2, observa-se que cerca de $80 \%$ dos pacientes tiveram suas mamas examinadas por um profissional de saúde antes de realizar a mamografia. 
Tabela 2 - Exames de mamografia de acordo com as variáveis dos exames, 2011-2021.

\begin{tabular}{|c|c|c|}
\hline Exame clínico anterior & Total & Percentual (\%) \\
\hline Sim & 12.689 .906 & 79,72 \\
\hline Não & 1.434 .187 & 9,01 \\
\hline Ignorado & 1.794 .820 & 11,27 \\
\hline \multicolumn{3}{|l|}{ Mamografia anterior } \\
\hline Sim & 11.636 .110 & 73,10 \\
\hline Não & 2.826 .980 & 17,76 \\
\hline Não sabe & 1.455 .823 & 9,14 \\
\hline \multicolumn{3}{|l|}{ Periodicidade } \\
\hline Mesmo ano & 549.188 & 3,45 \\
\hline 1 ano & 4.929 .269 & 31,00 \\
\hline 2 anos & 3.581 .660 & 22,45 \\
\hline 3 anos & 1.322 .671 & 8,32 \\
\hline 4 anos ou mais & 1.233 .656 & 7,75 \\
\hline Ignorado & 4.302 .469 & 27,03 \\
\hline \multicolumn{3}{|l|}{ Indicação clínica } \\
\hline Diagnóstico & 393.977 & 2,47 \\
\hline Rastreamento & 15.524 .936 & 97,53 \\
\hline \multicolumn{3}{|l|}{ Nódulo na requisição da mamografia } \\
\hline Não & 14.535 .736 & 91,31 \\
\hline Mama direita & 530.171 & 3,33 \\
\hline Mama esquerda & 574.136 & 3,61 \\
\hline Ambas as mamas & 278.870 & 1,75 \\
\hline \multicolumn{3}{|l|}{ Tipo de mamografia de rastreio } \\
\hline População alvo & 14.513 .435 & 91,20 \\
\hline População de risco elevado (história familiar) & 739.186 & 4,64 \\
\hline Paciente já tratado de câncer de mama & 272.295 & 1,71 \\
\hline \multicolumn{3}{|l|}{ Tempo entre solicitação e liberação do exame } \\
\hline Até 30 dias & 7.615 .127 & 47,80 \\
\hline 31 a 60 dias & 3.978 .193 & 25,00 \\
\hline Mais que 60 dias & 4.325 .593 & 27,20 \\
\hline \multicolumn{3}{|l|}{ Linfonodos axilares } \\
\hline Normais & 8.773 .538 & 55,11 \\
\hline Alterados & 115.203 & 0,72 \\
\hline Não visualizados & 4.248 .744 & 26,67 \\
\hline \multicolumn{3}{|l|}{ BI-RADS } \\
\hline Categoria 0 & 1.801 .209 & 11,32 \\
\hline Categoria 1 & 5.851 .100 & 36,76 \\
\hline Categoria 2 & 7.726 .629 & 48,54 \\
\hline Categoria 3 & 390.287 & 2,44 \\
\hline Categoria 4 & 119.287 & 0,75 \\
\hline Categoria 5 & 21.964 & 0,14 \\
\hline Categoria 6 & 7.847 & 0,05 \\
\hline
\end{tabular}

Fonte: Brito HRA, et al., 2021.

A indicação clínica para o exame de mamografia foi majoritariamente para o rastreamento, aproximadamente $98 \%$ dos exames. Além disso, cerca de $63 \%$ (9.776.926 pacientes) das mamografias solicitados com indicação para rastreamento de câncer de mama correspondiam a faixa etária de recomendação do Ministério da Saúde (50 a 69 anos). Ao levar em consideração a recomendação da FEBRASGO, do CBR e da SBM para isso, entre 40 e 74 anos, cerca de 93,7\% (14.553.466) dos exames de rastreio foram realizados na faixa etária indicada (Tabela 2 ).

Nota-se, a partir da Tabela 2, que o BI-RADS 2 foi a classificação mais frequentes durante o período avaliado, em cerca de $48,5 \%$ dos laudos. Já a Tabela 3, a qual contém a relação entre a categoria BI-RADS e a idade do paciente nos respectivos anos estudados, é possível observar uma linearidade entre as variáveis estudadas, por exemplo o percentual de exames laudados na categoria 0 varia entre 11 a $12 \%$, enquanto que na categoria 6 o percentual em todos os anos foi menor que $0,01 \%$. 
Tabela 3- Relação entre o exame de mamografia de acordo com a idade e a categoria BI-RADS, $2011-2021$.

\begin{tabular}{|c|c|c|c|c|c|c|}
\hline BI-RADS & $<20 a$ & $20-39$ a & $40-49 a$ & $50-69 a$ & $>70 a$ & Total n (\%) \\
\hline \multicolumn{7}{|c|}{2015} \\
\hline Categoria 0 & 265 & 10.508 & 77.377 & 125.425 & 11.957 & $225.532(11,0)$ \\
\hline Categoria 1 & 842 & 48.721 & 324.708 & 442.695 & 20.449 & $837.418(40,8)$ \\
\hline Categoria 2 & 486 & 24.593 & 219.445 & 592.007 & 85.201 & $921.741(44,9)$ \\
\hline Categoria 3 & 28 & 1.564 & 13.865 & 31.566 & 3.506 & $50.530(2,4)$ \\
\hline Categoria 4 & 9 & 683 & 3.821 & 7.647 & 1.453 & $13.613(0,66)$ \\
\hline Categoria 5 & 0 & 119 & 510 & 1.460 & 492 & $2.581(0,12)$ \\
\hline Categoria 6 & 0 & 68 & 147 & 357 & 94 & $666(0,03)$ \\
\hline \multicolumn{7}{|c|}{2016} \\
\hline Categoria 0 & 239 & 10.642 & 78.532 & 142.936 & 12.558 & $244.907(11,1)$ \\
\hline Categoria 1 & 748 & 45.990 & 316.379 & 486.021 & 22.374 & $871.514(39,5)$ \\
\hline Categoria 2 & 408 & 25.303 & 228.507 & 675.894 & 88.006 & 1.018.127 $(46,1)$ \\
\hline Categoria 3 & 35 & 1.698 & 14.519 & 36.401 & 3.641 & $56.294(2,5)$ \\
\hline Categoria 4 & 4 & 722 & 3.560 & 8.262 & 1.564 & $14.112(0,63)$ \\
\hline Categoria 5 & 3 & 135 & 463 & 1.583 & 477 & $2.661(0,12)$ \\
\hline Categoria 6 & 0 & 88 & 163 & 378 & 79 & $708(0,03)$ \\
\hline \multicolumn{7}{|c|}{2017} \\
\hline Categoria 0 & 264 & 11.562 & 85.487 & 178.216 & 14.662 & $290.192(11,0)$ \\
\hline Categoria 1 & 789 & 47.834 & 328.804 & 604.504 & 24.512 & $1.006 .445(38,1)$ \\
\hline Categoria 2 & 474 & 27.952 & 258.573 & 869.783 & 99.059 & $1.255 .846(47,6)$ \\
\hline Categoria 3 & 41 & 1.852 & 16.113 & 45.114 & 4.567 & $67.687(2,5)$ \\
\hline Categoria 4 & 7 & 873 & 4.042 & 10.416 & 1.769 & $17.107(0,65)$ \\
\hline Categoria 5 & 1 & 137 & 570 & 1.890 & 578 & $3.176(0,12)$ \\
\hline Categoria 6 & 0 & 102 & 223 & 423 & 159 & $854(0,03)$ \\
\hline \multicolumn{7}{|c|}{2018} \\
\hline Categoria 0 & 236 & 11.670 & 92.528 & 192.745 & 16.106 & $313.286(11,1)$ \\
\hline Categoria 1 & 725 & 46.020 & 348.759 & 607.418 & 26.421 & $1.029 .344(36,6)$ \\
\hline Categoria 2 & 554 & 29.790 & 293.297 & 944.742 & 107.949 & $1.376 .333(49,0)$ \\
\hline Categoria 3 & 47 & 1.729 & 15.862 & 45.432 & 4.608 & $67.678(2,4)$ \\
\hline Categoria 4 & 8 & 1.048 & 4.916 & 12.631 & 2.185 & $20.788(0,74)$ \\
\hline Categoria 5 & 1 & 177 & 635 & 2.163 & 682 & $3.658(0,13)$ \\
\hline Categoria 6 & 1 & 177 & 286 & 481 & 126 & $1.071(0,03)$ \\
\hline \multicolumn{7}{|c|}{2019} \\
\hline Categoria 0 & 274 & 12.462 & 101.116 & 209.360 & 18.045 & $341.455(11,1)$ \\
\hline Categoria 1 & 834 & 46.457 & 365.881 & 636.977 & 29.590 & $1.080 .411(35,2)$ \\
\hline Categoria 2 & 651 & 32.209 & 339.583 & 1.052 .601 & 118.379 & $1.544 .306(50,3)$ \\
\hline Categoria 3 & 50 & 1.819 & 16.885 & 48.486 & 4.878 & $72.137(2,4)$ \\
\hline Categoria 4 & 19 & 1.170 & 6.128 & 14.546 & 2.581 & $24.445(0,8)$ \\
\hline Categoria 5 & 1 & 242 & 830 & 2.474 & 793 & $4.341(0,14)$ \\
\hline Categoria 6 & 2 & 234 & 424 & 854 & 185 & $1.699(0,06)$ \\
\hline \multicolumn{7}{|c|}{2020} \\
\hline Categoria 0 & 174 & 7.861 & 68.550 & 138.048 & 11.707 & $226.340(12,1)$ \\
\hline Categoria 1 & 473 & 23.723 & 208.688 & 365.169 & 16.898 & $614.950(32,9)$ \\
\hline Categoria 2 & 178 & 20.937 & 220.578 & 643.704 & 69.902 & $955.480(51,1)$ \\
\hline Categoria 3 & 354 & 1.249 & 11.415 & 30.854 & 3.165 & $46.708(2,5)$ \\
\hline Categoria 4 & 25 & 990 & 4.245 & 10.298 & 1.878 & $7.505(0,4)$ \\
\hline Categoria 5 & 7 & 186 & 622 & 1.916 & 566 & $17.418(0,9)$ \\
\hline Categoria 6 & 0 & 262 & 5011 & 764 & 139 & $1.696(0,1)$ \\
\hline \multicolumn{7}{|c|}{2021} \\
\hline Categoria 0 & 101 & 5.243 & 49.817 & 96.729 & 7.601 & $159.497(12,5)$ \\
\hline Categoria 1 & 223 & 13.860 & 140.884 & 245.971 & 10.064 & $411.018(32,3)$ \\
\hline Categoria 2 & 228 & 13.684 & 161.156 & 436.341 & 43.350 & $654.796(51,5)$ \\
\hline Categoria 3 & 7 & 765 & 7.714 & 19.372 & 1.979 & $29.840(2,4)$ \\
\hline Categoria 4 & 5 & 672 & 2.961 & 6.860 & 1.295 & $11.794(0,9)$ \\
\hline Categoria 5 & 2 & 134 & 431 & 1.277 & 413 & $2.257(0,2)$ \\
\hline Categoria 6 & 0 & 197 & 299 & 524 & 133 & $1.153(0,1)$ \\
\hline Total $\mathbf{n}$ & 9.823 & 536.113 & 4.445 .309 & 10.031 .715 & 898.775 & 15.923 .115 \\
\hline Total \% & 0,06 & 3,37 & 27,92 & 63,00 & 5,65 & 100 \\
\hline Média & 200,47 & 10.941 & 90.720 & 204.728 & 18.342 & - \\
\hline Amplitude & 842 & 48.653 & 365.734 & 1.052 .244 & 118.300 & - \\
\hline Desvio padrão & 262 & 15.173 & 121.171 & 288.192 & 30.530 & - \\
\hline
\end{tabular}

Fonte: Brito HRA, et al., 2021. 
No Gráfico 1 é possível observar uma queda brusca no número de mamografias realizadas no ano de 2020, ao comparar com o ano anterior houve uma diminuição em que aproximadamente $61 \%$ do número de exames realizados.

Gráfico 1 - Número de mamografias realizadas por ano.

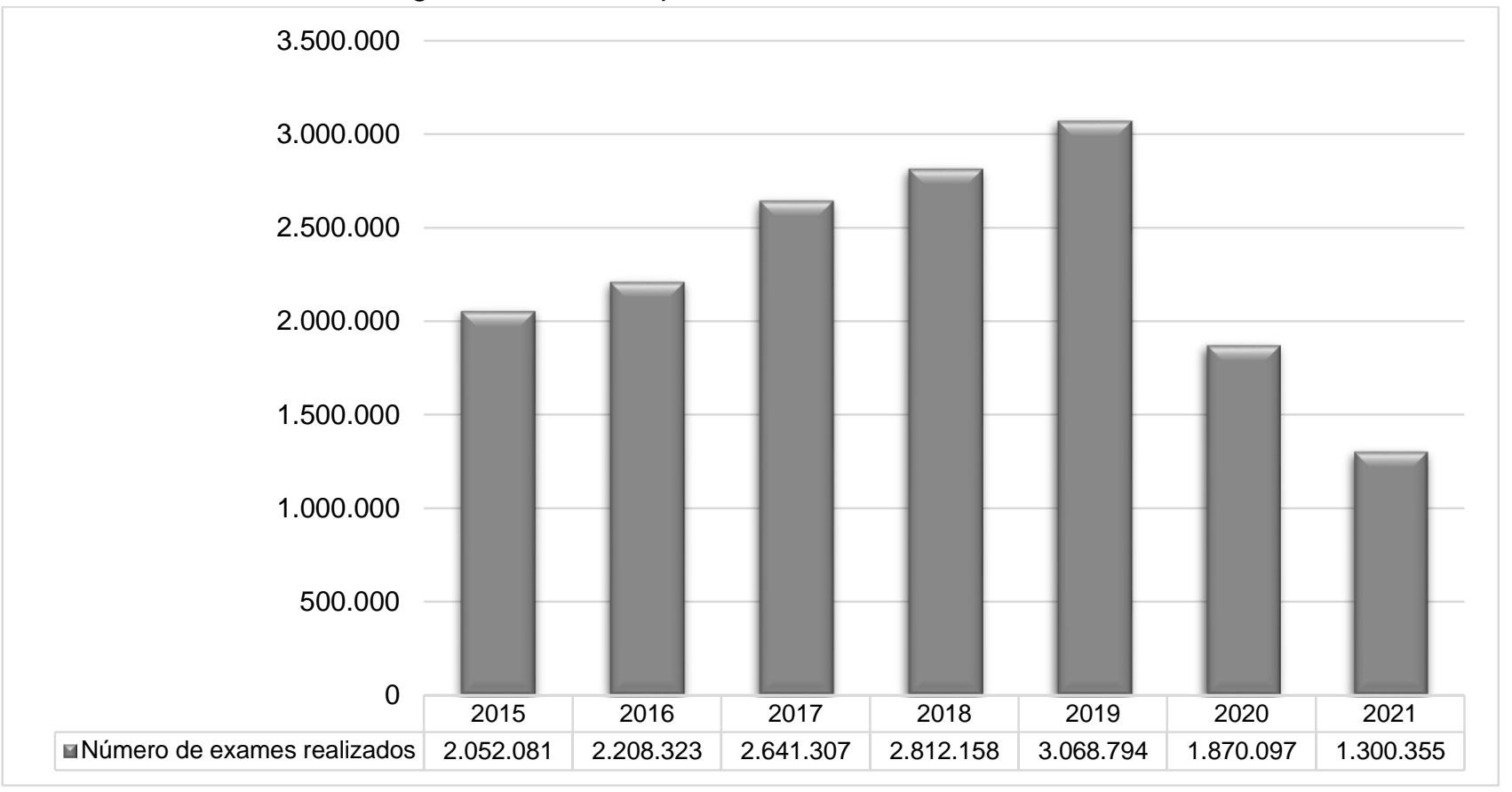

Fonte: Brito HRA, et al., 2021.

\section{DISCUSSÃO}

É importante pontuar que ao longo das últimas décadas, desde 1970, as taxas de mortalidade por câncer de mama têm diminuído devido à melhora no rastreamento do câncer de mama e as melhorias na terapia adjuvante (BOONIE CY, et al., 2021). As pesquisas radiológicas para desenvolver a melhor técnica de avaliação das mamas iniciaram a partir de 1913 quando o cirurgião Albert Salomon reportou achados radiográficos de espécimes de mastectomia identificando os variados aspectos nodulares e sua correlação anatomopatológica. Desde então, a mamografia tornou-se uma forma particular de radiografia que trabalha com níveis de radiação entre intervalos específicos com a finalidade de registrar imagens da mama para diagnosticar a eventual presença de estruturas que indiquem uma doença, com especial importância para o câncer (KOCH H, 2016).

Entre 2015 e 2021, houve registros de 15.923 .115 exames de mamografia, sendo que $0,19 \%$ foram realizados no sexo masculino, o baixo números de mamografias nesse grupo pode ser explicado pelo fato do câncer de mama acometer raramente os homens, representando menos de $1 \%$ de todos os cânceres que os acometem (INCA, 2021; SALOMON MFB, et al., 2015). Ademais, sabe-se que a estratégia de prevenção do câncer de mama visa uma abordagem populacional, por tratar-se de um programa de rastreamento para as mulheres na faixa etária e periodicidade recomendadas, no caso do sexo masculino a prevenção teria caráter individual, de acordo com os fatores de risco (MS, 2010). Em consequência de fatores fisiológicos e ambientais envolvidos, sabe-se que as mulheres têm maior risco de desenvolverem câncer de mama, por isso $99,81 \%$ das mamografias foram realizadas nelas (SALOMON MFB, et al., 2015).

É válido mencionar que o exame mamográfico é realizado nos homens quando há sinais e sintomas locais (secreção papilar normalmente sanguinolenta, nódulo palpável e outros), mas por se tratar de uma doença mais comum nas mulheres, muitos homens não sabem da possibilidade de desenvolver o câncer de mama e, consequentemente, não percebem esses sinais da doença retardando seu diagnóstico. Logo, na maioria dos casos, o diagnóstico é feito tardiamente, em estádios mais avançados, o que compromete o prognóstico 
e acarreta maior morbimortalidade em relação aos casos de neoplasia mamária feminina. Sendo assim, um fator que provavelmente contribui para que as taxas de mortalidade por neoplasia mamária mantenham-se elevadas é o diagnóstico tardio, em estádios avançados (KOMEN SG, 2010; GONÇALVES CV, et al., 2017).

Ademais, levando em consideração fatores de risco e predisponentes ao câncer de mama como obesidade, tecido mamário denso, menarca precoce, menopausa posterior, nuliparidade, aumento da idade na primeira gravidez e hábitos de vida como tabagismo e uso de álcool; destaca-se o fator de risco primário mais prevalente: idade avançada. Mulheres, sobretudo a partir dos 50 anos de idade, têm maior risco de desenvolver câncer de mama. Em 2016, aproximadamente 99,3\% e 71,2\% de todas as mortes associadas ao câncer de mama na América foram relatadas em mulheres com mais de 40 e 60 anos, respectivamente (WORLD HEALTH ORGANIZATION (WHO), 2018; SUN YG, et al., 2017; COSTA LS, et al., 2021).

Observa-se que $63 \%$ das mamografias foram feitas entre 50 e 69 anos, faixa etária recomendada pelo MS, enquanto que cerca de $28 \%$ entre 40 e 49 , intervalo de idade preconizado pelo CBR e pela SBM. Além disso, no que diz respeito a periodicidade, aproximadamente $53 \%$ das mamografias foram realizadas no intervalo de 1 a 2 anos, a qual condiz com a indicação da FEBRASGO (anual) ou do MS (bianual); é importante salientar que, na pesquisa, $27 \%$ ignoraram essa periodicidade.

Outrossim, em um estudo realizado na Suécia, uma redução da mortalidade por câncer de mama de 26$29 \%$ foram encontradas para mulheres que foram diagnosticadas por rastreamento entre 40 e 49 anos. Sendo que, uma metanálise de oito ensaios mostrou uma redução significativa de $15 \%$ na mortalidade por câncer de mama em mulheres entre 39 anos e 49 anos. A mamografia é um passo fundamental para essa redução, visto que possibilita o diagnóstico precoce da doença (GELDER L, et al., 2015).

Sabe-se que realização do primeiro exame de mamografia de forma precoce está associado a fatores como maior nível de escolaridade, menopausa e obesidade, entretanto nesse estudo há uma dificuldade em relacionar o nível de escolaridade com a execução da mamografia, visto que cerca de $99,8 \%$ dos casos não tiveram o item referente a escolaridade preenchido (SEDIYAMA CMNO, et al., 2021). Observa-se que uma adesão de aproximadamente $80 \%$ a realização do exame clínico das mamas no período avaliado, mas é importante ressaltar que o exame clínico e o autoexame da mama não excluem a necessidade de realizar a mamografia (INCA, 2017).

Sabe-se da importando das alterações dos linfonodos axilares no câncer de mama, visto que é o fator prognóstico isolado de maior relevância no exame, em que a presença de metástases axilares é um fator independente que mostra maior agressividade do tumor, havendo relação com o prognóstico do paciente. Ressalta-se que linfonodos sem alterações são pequenos e podem ser difíceis de serem diagnosticados, mas quando há presença de um processo infeccioso, inflamatório ou neoplásico, uma das alterações que pode acontecer é o aumento do tamanho do linfonodo, por exemplo quando a neoplasia se dissemina para o interior dos gânglios linfáticos (FEBRASGO, 2010).

Comprovadamente a mamografia não tem acurácia adequada para identificar metástases em linfonodos axilares, dado isso a avaliação linfonodal é preconizada através do uso da ultrassonografia quando há alterações de linfonodos no exame físico (INCA, 2019b; PINHEIRO DJP, et al., 2014). Atualmente, a tomossíntese digital mamária encontra-se em evidência devido a sua capacidade de permitir a visualização tridimensional da mama, consequentemente, havendo redução das limitações da mamografia, como a sobreposição de tecidos ou as dificuldades relacionadas com a densidade mamária (ARDISSON MD, et al., 2021).

No que diz respeito a classificação BI-RADS, a análise foi similar observa-se que a categoria 1 e 0 é mais frequente em indivíduos mais jovens, à medida que a idade aumenta, há uma mudança para as categorias 2 e 3. Por fim, as categorias 4 e 5 são mais frequentes após 70 anos de idade, similar ao observado em outros estudos como Tomazelli JG, et al. (2017). Isso também pode ser explicado pelo fato da incidência do câncer de mama aumentar com a idade, além do fato da mama ser densa em jovens, limitando a aplicabilidade da mamografia para detecção de lesões. É válido mencionar que cerca de $85,5 \%$ dos exames foram laudados na categoria 1 e 2, logo a conduta é apenas o acompanhamento (CBR, 2016; MS, 2018). 
Outro ponto importante observado no estudo foi a queda do número de mamografias realizadas em 2020, em que uma redução em que aproximadamente $61 \%$ do número de exames realizados no ano anterior. Isso é explicado pela suspensão dos procedimentos eletivos, incluindo rastreamento de câncer, além das medidas de isolamento social e das paralisações de várias atividades durante a pandemia da doença do coronavírus (COVID-19), de modo que fosse possível priorizar as urgências/emergênciais e reduzir a transmissão da doença. Ressalta-se que o rastreamento de neoplasias durante a pandemia necessita de uma análise sobre o cenário epidemiológico local, além de uma avaliação dos riscos e dos benefícios (INCA, 2020b, MIGOWSKI A e CORRÊA FM, 2020). Outros estudos observaram a redução da realização da mamografia preventiva durante o período de pandemia, sendo que houve um decréscimo anual entre $8 \%$ a $47 \%$ avaliado pelos autores (VANNI G, et al., 2020; BESSA JF, 2021; FEDEWA AS, et al., 2021; MILLER MM, et al., 2021; TSAI HY, et al., WALKER MJ, et al., 2021).

\section{CONCLUSÃO}

Observou-se que o a maioria das mamografias foram classificadas na categoria 2 do BI-RADS, logo apresentavam achados benignos, além de que a principal indicação do exame foi para rastreio do câncer de mama de acordo com as indicações do MS e da FEBRASGO, ressalta-se que a maioria dos exames foram realizados nas idades preconizadas por essas instituições. Além disso, houve uma diminuição significativa no número de mamografias realizadas durante o ano de 2020 devido a pandemia pelo SARS-CoV-2. Este estudo é extremamente relevante, visto que é necessário avaliar o rastreamento de determinada neoplasia em uma região, para que seja possível analisar se o número de exames cresce ou não proporcionalmente com a faixa etária em que deve ser solicitado, avaliando os fatores relacionados com essas alterações.

\section{REFERÊNCIAS}

1. ARDISSON MD, et al. Avanços no rastreamento mamográfico e o manejo daspacientes com câncer de mama. Revista Eletrônica Acervo Saúde, 2021; 13(1): e5630.

2. BESSA JF. Breast imaging hindered during covid-19 pandemic, in Brazil. Revista de Saúde Pública; $2021,55$.

3. Bonnie CY. Epidemiology of breast cancer in young women. Breast disease, 2005; 23(1): 3-8.

4. COLÉGIO AMERICANO DE RADIOLOGIA. ACR BI-RADS® Atlas Fifth Edition: quick reference. Estados Unidos; 2021. Disponível em: https://www.acr.org/-/media/ACR/Files/RADS/BI-RADS/BIRADS-Reference-Card.pdf. Acessado em: 28 de setembro de 2021.

5. COLÉGIO BRASILEIRO DE RADIOLOGIA E DIAGNÓSTICO POR IMAGEM (CBR). Comissão Nacional de Mamografia - Colégio Brasileiro de Radiologia e Diagnóstico por Imagem (CBR), Sociedade Brasileira de Mastologia (SBM) e Federação Brasileira das Associações de Ginecologia e Obstetrícia (FEBRASGO). São Paulo; 2020. Disponível em: http://www.spmastologia.co m.br/diagnostico-por-imagem/rastreamento. Acessado em: 15 de setembro de 2021.

6. COLÉGIO BRASILEIRO DE RADIOLOGIA E DIAGNÓSTICO POR IMAGEM (CBR). BI-RADS - Sistema de laudos e registro de dados de imagem da mama. São Paulo: Colégio Brasileiro de Radiologia, 2005.

7. COLÉGIO BRASILEIRO DE RADIOLOGIA E DIAGNÓSTICO POR IMAGEM (CBR). Atlas BI-RADS do ACR: sistema de laudos e registro de dados de imagem da mama/ American College of Radiology. 2 ed. São Paulo: Colégio Brasileiro de Radiologia, 2016.

8. COSTA LS, et al. Fatores de risco relacionados ao câncer de mama e a importância da detecção precoce para a saúde da mulher. Revista Eletrônica Acervo Científico, 2021, 31; e8174.

9. FEDERAÇÃO BRASILEIRA DAS ASSOCIAÇÕES DE GINECOLOGIA E OBSTETRÍCIA (FEBRASGO). Manual de

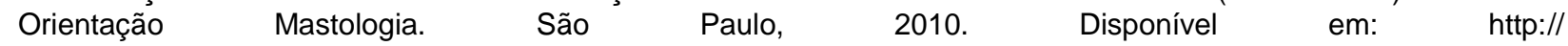
professor.pucgoias.edu.br/SiteDocente/admin/arquivosUpload/13162/material/MASTOLOGIA\%20\%20FEBRASGO\%202010.pdf. Acessado em: 15 de setembro de 2021.

10. FEDEWA SA, et al. Changes in breast cancer screening rates among 32 community health centers during the COVID19 pandemic. Cancer, 2021.

11. GELDER L, et al. Magnetic resonance imaging in patients with unilateral bloody nipple discharge: useful when conventional diagnostic are negative? World J Surg. 2015; 39: 184-6.

12. GONÇALVES CV, et al. O conhecimento de mulheres sobre os métodos para prevenção secundária do câncer de mama. Ciênc. saúde colet. 2017; 22 (12).

13. INSTITUTO NACIONAL DE CÂNCER (INCA). A situação do câncer de mama no Brasil: Síntese de dados dos sistemas de informação. Rio de Janeiro, 2019a. Disponível em: https://www.inca.gov.br/sites/ufu.sti.inca.local/files/media/document/a_situacao_ca_mama_brasil_2019.pdf. Acessado em 25 agosto 2021. 
14. INSTITUTO NACIONAL DE CÂNCER (INCA). Atualização em mamografia para técnicos em radiologia: para técnicos em radiologia. 2. ed. Rio de Janeiro, 2019b. Disponível em: https://www.inca.gov.br/sites/ufu.sti.inca.local/files/media/document/2a_edicao_atualizacao_em_mamografia_para_t ecnicos_em_radiologia_2019.pdf. Acessado em: 27 setembro 2021.

15. INSTITUTO NACIONAL DE CÂNCER (INCA). Câncer de mama, 2021. In: Instituto Nacional de Câncer. Brasil, 2021. Disponível em: https://www.inca.gov.br/tipos-de-cancer/cancer-de-mama. Acesso em: 25 agosto 2021.

16. INSTITUTO NACIONAL DE CÂNCER (INCA). Diretrizes para a detecção precoce do câncer de mama no Brasil: Sumário executivo. Rio de Janeiro, 2017.

17. INSTITUTO NACIONAL DE CÂNCER (INCA). Estimativa 2020. Brasil, 2020a. Disponível em: https://www.inca.gov.br/estimativa/introducao. Acessado em: 25 agosto 2021.

18. INSTITUTO NACIONAL DE CÂNCER (INCA). Nota Técnica - DIDEPRE/CONPREV/INCA Rastreamento de câncer durante a pandemia de COVID-19 09/07/2020, 2020. Brasil, 2020b. Disponível em: https://www.inca.gov.br/sites/ufu.sti.inca.local/files/media/document/nota-tecnica-rastreamento-covid-didepre-09-jul2020.pdf. Acessado em 2 setembro 2021.

19. INSTITUTO NACIONAL DE CÂNCER (INCA). Parâmetros para o rastreamento do câncer de mama: recomendações para gestores estaduais e municipais. Rio de Janeiro, 2009. Disponível em: https://bvsms.saude.gov.br/bvs/publicacoes/parametros_rastreamento_cancer_mama.pdf. Acessado em 25 agosto 2021.

20. INSTITUTO NACIONAL DE CÂNCER (INCA). Sistema BI-RADS: condutas. Rio de Janeiro, 2018. Disponível em: https://www.arca.fiocruz.br/bitstream/icict/29924/2/SISTEMA\%20BI-RADS_CONDUTAS.pdf. Acessado em 28 setembro 2021.

21. KOCH H. Mamografia como método para diagnosticar o câncer de mama. Radiol Bras; $2016 ; 49$ (6).

22. KOMEN SG. Câncer de mama em homens. Facts for life. Estados Unidos, 2010. Disponível em: https://ww5.komen.org/uploadedFiles/Content_Binaries/translate/Breast\%20Cancer\%20in\%20Men_portuguese.pdf. Acessado em: 25 agosto 2021.

23. MIGOWSKI A, CORRÊA FM. Recomendações para detecção precoce de câncer durante a pandemia de covid-19 em 2021. Rev. APS, 2020; 23 (1): $235-240$

24. MIGOWSKI A, et al. Diretrizes para detecção precoce do câncer de mama no Brasil. II - Novas recomendações nacionais, principais evidências e controvérsias. Cad. Saúde Pública, 2018; 34(6): e00074817.

25. MILLER MM, et al. Impact of the COVID-19 pandemic on breast cancer screening volumes and patient screening behaviors. Breast cancer research and treatment, 2021: 1-10.

26. MINISTÉRIO DA SAÚDE (MS). Fundação Oswaldo Cruz. Sistema BI-RADS: condutas. Brasília, 2018. Disponível em: https://www.arca.fiocruz.br/bitstream/icict/ 29924/2/SISTEMA\%20BI-RADS_CONDUTAS.pdf. Acessado em: 25 agosto 2021.

27. MINISTÉRIO DA SAÚDE (MS). Secretaria de Atenção à Saúde. Departamento de Atenção Básica. Rastreamento: Série A. Normas e Manuais Técnicos Cadernos de Atenção Primária, n. 29. Brasília, 2010. Disponível em: https://bvsms.saude.gov.br/bvs/publicacoes/caderno_atencao_primaria_29_rastreamento.pdf. Acessado em: 25 agosto 2021.

28. PINHEIRO DJP, et al. Linfonodos axilares em pacientes com câncer de mama: avaliação ultrassonográfica. Radiol Bras, 2014; 47 (4).

29. SALOMON MFB, et al. Câncer de mama no homem. Rev. Bras. Mastologia., 2015; 25(4): 141-5.

30. SEDIYAMA CMNO, et al. Fatores relacionados à idade de realização do primeiro exame de mamografia em mulheres atendidas em um serviço público de Belo Horizonte -MG. Revista Eletrônica Acervo Saúde, 2021; $13(5)$ : e7497.

31. SUN YS, et al. Risk Factors and Preventions of Breast Cancer. Int J Biol Sci. 2017;13(11): 1387-1397.

32. SOUSA CNS, et al. Rastreamento do câncer de mama: conhecimentos e práticas de trabalhadores na Unidade Básica de Saúde. REFACS, 2019; 7 (3).

33. TOMAZELLI JG, et al. Rastreamento do câncer de mama no Brasil: uma avaliação da oferta e utilização da rede assistencial do Sistema Único de Saúde no período 2010-2012*. Epidemiol. Serv. Saúde, 2017; 26(4).

34. TSAI HY, et al. Effects of the COVID-19 pandemic on breast cancer screening in Taiwan. The Breast; $2020,54: 52-55$.

35. URBAN L, et al. Recomendações do Colégio Brasileiro de Radiologia e Diagnóstico por Imagem, da Sociedade Brasileira de Mastologia e da Federação Brasileira das Associações de Ginecologia e Obstetrícia para rastreamento do câncer de mama por métodos de imagem*. Radiol Bras., 2012; 45(6): 334-339.

36. VANNI G, et al. Lockdown of Breast Cancer Screening for COVID-19: Possible Scenario. In Vivo, 2020, 34(5): 30473053.

37. WALKER MJ, et al. Measuring the impact of the COVID-19 pandemic on organized cancer screening and diagnostic follow-up care in Ontario, Canada: A provincial, population-based study. Preventive Medicine, 2021, 151: 106586.

38. WORLD HEALTH ORGANIZATION (WHO). Health topics. Breast cancer: prevention and control. Geneva, 2020. Disponível em: https://www.who.int/cancer/detection/breastcancer/en/. Acessado em: 27 agosto 2021. 